\title{
Ferroptosis involves in intestinal epithelial cell death in ulcerative colitis
}

\author{
Minyi Xu', Jin Tao', Yidong Yang ${ }^{1}$, Siwei Tan', Huiling Liu', Jie Jiang ${ }^{1}$, Fengping Zheng ${ }^{1}$ and Bin $\mathrm{Wu}^{1}$
}

\begin{abstract}
Ferroptosis has recently emerged as an iron-dependent form of nonapoptotic cell death, which is also a regulated necrosis process and a response to tumor suppression. However, whether ferroptosis is involved in ulcerative colitis (UC) is unknown. The aims of this study were to investigate whether the ferroptosis is involved in UC, particularly intestinal epithelial cell (IEC) death, and to analyze the effect of the nuclear factor kappa Bp65 subunit (NF-kBp65) on ferroptosis. The gene expression of ferroptosis-related proteins was assessed in intestinal mucosal samples from human UC. The experimental model of UC was induced with dextran sulfate sodium (DSS). Ferroptosis of IECs was evaluated, the effect of NF-KBp65 on ferroptosis was analyzed by using IEC-specific NF-KBp65-deleted mice (p65 ${ }^{\mathrm{IEC}-\mathrm{KO}}$ ), and the ferroptosis signaling pathway was investigated in vitro and in vivo. The results showed that ferroptosis was significantly induced in the IECS from UC patients and mice with colitis, and the ferroptosis was mediated by endoplasmic reticulum (ER) stress signaling. The specific deletion of IEC NF-KBp65 clearly upregulated ferroptosis and exacerbated colitis, and the result showed that phosphorylated-NF-kBp65 significantly inhibited ER stress signaling by directly binding eukaryotic initiation factor $2 a$. These data indicate that ferroptosis contributes to UC via ER stressmediated IEC cell death, and that NF-kBp65 phosphorylation suppresses ER stress-mediated IEC ferroptosis to alleviate UC. The results suggest that ferroptosis involves in IEC death in UC, NF-KBp65 play a critical role in the ferroptotic inhibition, and ferroptosis is a potential therapeutic target for UC.
\end{abstract}

\section{Introduction}

Ulcerative colitis (UC) is a chronically relapsing inflammatory disease of the colon and rectum, which is clinically characterized by abdominal pain, rectal bleeding, and diarrhea ${ }^{1}$. Although not fully understood, the etiology of $\mathrm{UC}$ is generally thought to be genetically based with contributions from gut microbial factors and a disordered immune response ${ }^{2}$. Recent studies have revealed the main immunological/inflammatory mechanisms of UC, and have provided new methods for modulating inflammation, but few have explored the biological changes in colonic intestinal epithelial cells (IECs).

Ferroptosis has recently emerged as a newly discovered form of regulated necrosis, and it presents features that

\footnotetext{
Correspondence: Bin Wu (wubin6@mail.sysu.edu.cn)

'Department of Gastroenterology, The Third Affiliated Hospital of Sun Yat-Sen University, Guangzhou, China

These authors contributed equally: Minyi Xu and Jin Tao

Edited by A. Stephanou
}

distinguish it from other kinds of cell death at the cell morphology, biochemistry, and genetics levels ${ }^{3,4}$. Shrunken mitochondria; condensed dense mitochondrial membranes; and reduced mitochondrial crista are the main morphological features of ferroptosis, ${ }^{3,4}$. Iron accumulation and increased reactive oxygen species (ROS) production from lipid peroxidation play crucial roles in triggering ferroptosis $^{3}$. In addition, downregulation of glutathione peroxidase 4 (GPX4), a scavenger of lipid ROS and increased mRNA levels of prostaglandin-endoperoxide synthase 2 (PTGS2) are key characteristics of ferroptotic cell death ${ }^{5}$. Although its biological function and molecular mechanism have not been thoroughly elucidated, ferroptosis has been implicated in multiple diseases ${ }^{5-8}$. Interestingly, in previous studies by different groups, the administration of iron chelator was found to significantly reduce ROS in colonic tissues of UC patients and to ameliorate clinical symptoms and improve endoscopic presentations ${ }^{9,10}$. In contrast, high dietary iron supplements exacerbated UC symptoms in both patients 
and murine models ${ }^{11,12}$. These findings indicated the important effects of iron and ROS on the pathogenesis of UC. However, whether iron- and ROS-triggered ferroptosis involves in UC remains unclear.

Endoplasmic reticulum (ER) stress has long been considered a promoting factor in UC. Emerging evidence has shown that ER stress signaling has close cross-talk with ferroptosis. Protein kinase-like endoplasmic reticulum kinase (PERK) is a major sensor of ER stress. In response to ER stress, PERK is activated through phosphorylation, and then activates and governs the downstream signaling of the eukaryotic initiation factor $2, \alpha$ subunit (eIF2 $\alpha) /$ activating transcription factor 4 (ATF4)/C/EBP-homologous protein (CHOP) pathway ${ }^{13}$. PERK/eIF2 $\alpha$ signaling was found to regulate the production of ROS during ferroptosis ${ }^{14-16}$. It was reported that the activation of the PERK branch facilitated whole cigarette smoke condensate-induced ferroptosis in human bronchial epithelial cells ${ }^{14}$. Results from our recent study demonstrated that ER stress signaling promotes colonic epithelial apoptosis in $\mathrm{UC}^{17}$. However, the role of ER stressmediated ferroptosis in UC remains poorly understood and needs to be further investigated.

The transcription factor nuclear factor kappa B (NF-kB) has been confirmed to exert principal function on the modulation of UC. NF- $\mathrm{kB}$ family is composed of five members and the activity of NF-kB is mainly initiated by the phosphorylated subunit p65. Recently, numerous studies reported that the effects of activated NF- $\mathrm{kB}$ are cell typespecific. NF- $\mathrm{KB}$ promotes cytokine and chemokine production in inflammatory cells ${ }^{18}$, whereas, it also functions in IECs to maintain physiological homeostasis and contribute to damage resistance ${ }^{19}$. Our previous study revealed that the application of the NF- $\mathrm{KB}$ inhibitor BAY 11-7082 enhanced epithelial apoptosis in experimental colitis ${ }^{20}$, suggesting a role for activated NF- $\mathrm{kB}$ in inhibiting apoptosis. Moreover, growing evidence has indicated that NF- $\mathrm{kB}$ is involved in the regulation of ER stress signaling and ferroptosis process ${ }^{21,22}$. However, the definite protective mechanisms of NF- $\mathrm{kB}$ via the regulation of ER stress-mediated ferroptosis in UC are not completely understood.

In our present study, the effect and mechanism of ferroptosis in UC via the regulation of NF-KBp65/ER stress were investigated. We report first that ferroptosis is involved in the IEC cell death of UC that is mediated by ER stress signaling. Specifically, we found that phosphorylated-NF-kBp65 protects the IECs against ferroptosis by suppressing ER stress, suggesting that ferroptosis and NF-kBp65 phosphorylation are potential therapeutic targets for UC treatment.

\section{Materials and methods}

\section{Human colonic tissue samples}

Colonic mucosal specimens from patients with active UC (Mayo endoscopic score $\geq 2$ ) ) $^{23}$ and healthy controls were collected from the Gastroenterology Department of the Third Affiliated Hospital of Sun Yat-Sen University. Written informed consent was signed by each study participant. The acquisition of these samples was approved by the Clinical Research Ethics Committee of The Third Affiliated Hospital of Sun Yat-Sen University ([2018] 02-409-01).

\section{Microarray experiment}

Three paired colonic mucosal samples from colitis patients and control individuals were collected. Total RNA from each sample was extracted and reverse transcribed into cDNA. The cDNA was then processed for gene expression microanalysis as previously described ${ }^{24}$. The Gene Expression Omnibus (GEO) accession number from NCBI is GSE134025.

\section{Animal experiments}

All animal experiments in this study were conducted with the approval of the Institutional Animal Care and Use Committee at Sun Yat-Sen University. All mice involved had a C57BL/6 gene background. Wild-type mice were obtained from the Laboratory Animal Center of Guangdong Province. Mice carrying the loxP-flanked $N F-\kappa B p 65$ allele $\left[N F-\kappa B p 65^{f l o x / f l o x}\right]$ were precious gifts from Dr. Jianping Ye [Pennington Biomedical Research Center, Louisiana State University System, Baton Rouge, LA, USA]. Villin-cre transgenic mice were purchased from The Jackson Laboratory. Mice with IEC-specific NF$\kappa B p 65$-deletion $\left[\mathrm{p} 65^{\mathrm{IEC}-\mathrm{KO}}\right.$ ] were generated by breeding $N F-\kappa B p 65^{\text {flox/flox }}$ mice with villin-cre transgenic mice, and the $N F-\kappa B p 65^{f l o x / f l o x}$ littermates were used as wild-type (WT) mice. All mice were housed in rooms under controlled condition, with room temperature and with $50 \%$ humidity and 12-hour light-dark cycles. All mice with age- and sex-matched between 6 and 8 weeks of age were assigned randomly to groups. To induce experimental colitis, the mice were challenged with $3 \%$ dextran sulfate sodium (DSS; MP Biomedicals, LLC, Solon, OH) in drinking water for 7 days. The control mice were allowed to drink water only at the same time. To administer ferrostatin-1 (Fer1) in vivo, we intraperitoneal injected mice daily with Fer1 (Merck, Darmstadt, Germany, $2.5 \mu \mathrm{mol} / \mathrm{kg}$ body weight $)^{25}$, and the corresponding control mice were injected intraperitoneally with normal saline. To treat mice with GSK2606414 (GSK 414) in vivo, the mice were administered either GSK 414 (Selleck, Shanghai, China, suspended in vehicle solution containing $0.5 \%$ hydoxypropylmethyl cellulose and $0.1 \%$ Tween 80 in water at $\mathrm{pH} 4.8,50 \mathrm{mg} / \mathrm{kg}$ body weight ${ }^{26,27}$ or vehicle solution by oral gavage daily during DSS administration.

\section{Cell culture and drug treatment}

The HCoEpiC cell (human normal colonic epithelial cell) was cultured in colonic epithelial cell medium 
(CoEpiCM, ScienCell Research Laboratory, CA, USA) containing $10 \%$ fetal bovine serum and other supplements according to the manufacturer's instructions (ScienCell Research Laboratory). To induce ferroptosis, cells were seeded on 12-well plates and treated with RSL3 (Selleck, $20 \mu \mathrm{M})$ for 8 hours after plating. For the ER stress suppression experiment, $1 \mu \mathrm{M}$ GSK 414 was added to the medium 30 minutes before RSL3 challenge. For the TNF$\alpha$ treatment experiment, the cells were administrated with TNF- $\alpha$ (PeproTech, Rocky Hill, NJ, USA, $40 \mathrm{ng} / \mathrm{ml}$ ) for 1 hour and the NF-kB inhibitor, BAY 11-7085 (Merck, $10 \mu \mathrm{M}$ ), was added to some wells 15 minutes before the TNF- $\alpha$ was added.

\section{Statistical analysis}

All data were expressed as the means \pm SEM. Data were statistically analyzed by SPSS 22.0. Differences in two groups were analyzed using Student's $t$ test. Comparisons of multiple groups were analyzed by one-way ANOVA analysis of variance followed by post hoc Bonferroni correction. Differences were considered statistically significant when $P<0.05$. Unless indicated otherwise in the figure legends, all experimental results were based on $n=$ 6 mice or human samples per group, and all experiments were repeated twice. Information on the additional materials and methods used in this study can be found in the Supplementary materials and methods.

\section{Results}

\section{Ferroptosis was induced in UC in both human and mice}

In recent decades, multiple studies have revealed contributing roles of ROS and iron in the progression of $\mathrm{UC}^{28,29}$. Because ROS accumulation and iron overload are critical steps in the initiation of ferroptosis, we hypothesized that ferroptosis is implicated in UC. To verify this hypothesis, we collected colonic mucosal specimens from three UC patients and three control individuals to perform a gene expression microanalysis. Among the mRNA transcriptomes revealed through this detection, dozens of genes that had been previously identified as highly expressed in UC were also elevated in our data (Fig. 1a and Supplementary table 1$)^{30,31}$. Interestingly, several reported ferroptosis-associated genes, namely, acyl-CoA synthetase family member 2 (ACSF2), GPX4, lysophosphatidylcholine acyltransferase 3 (LPCAT3), nuclear receptor coactivator 4 (NCOA4), acyl-CoA synthetase long-chain family member 4 (ACSL4), solute carrier family 38, member 1 (SLC38A1), and glucose-6phosphate dehydrogenase (G6PD), all of which participate in the regulation of lipid or iron metabolism ${ }^{3,4,32}$, were remarkably downregulated or upregulated in the UC specimens in our study (Fig. 1a, b). This gene analysis indicated that ferroptosis may be involved in colitis. In addition, the clinical UC samples presented with broken epithelium, damaged crypts, and inflammatory cell infiltrations, findings that were histologically consistent with the characteristics of UC (Fig. 1c).

To confirm that ferroptosis is involved in colitis, we then measured MDA levels and iron contents in the colonic tissues. Compared with the control samples, the colitis specimens had significantly higher MDA and iron levels, particularly ferrous iron. (Fig. 1d, e). Ferritin is a cellular iron storage protein composed of two similar polypeptide chains: ferritin light chain (FTL) and ferritin heavy chain (FTH). Existing researches have shown that increased cellular iron levels during ferroptosis induce transcriptional upregulation of ferritin ${ }^{14,32,33}$. Indeed, we found that both the mRNA and protein levels of FTL and FTH were obviously increased in the UC tissues (Fig. 1f-h). Immunofluorescent assay revealed that the elevated positive signals for FTH were primarily evident in the epithelial cells (Fig. 1i), suggesting that ferroptosis mainly occurred in the epithelial cells. Moreover, we observed shrunken mitochondria in the UC samples by transmission electron microscopy (Fig. 1j), a finding consistent with the morphological characteristic of ferroptosis.

In light of the results described above for human UC, we induced experimental colitis in mice by challenging them with 3\% DSS for 7 days. DSS administration led to inflammatory damage in the colonic epithelium and abundant leukocytes, which were particularly evident with the neutrophil infiltration in the mucosa (Fig. 2a, b). Ferroptosis is a kind of programmed necrotic cell death, which can be identified through propidium iodide (PI) staining. Herein, we injected PI solution into mice before killed. The extracted colonic IECs were subject to flow cytometry to detect PI-positive cells. We found that the number of PI-positive IECs was increased in colitis mice (23.9\% in the DSS group vs $4.59 \%$ in the vehicle group) (Fig. 2c). Consistent with the data on human UC, colonic IECs from the DSS-treated mice had shrunken mitochondria and reduced mitochondrial crista (Fig. 2f), and with elevated levels of intracellular iron and ROS as well as upregulated FTL and FTH (Fig. 2d, e, g, h). Moreover, we observed increased PTGS2 and decreased GPX4, which were generally used as biomarkers of ferropto$\mathrm{sis}^{3,4,32}$, in the colonic IECs from experimental colitis mice, compared with the vehicle mice (Fig. 2i). These data indicated that the colonic epithelial cells of the DSStreated mice underwent ferroptosis.

On the basis of the findings described above, we concluded that ferroptosis was induced in the colon, predominately in the colonic IECs of UC.

\section{Inhibition of ferroptosis alleviated experimental colitis in mice}

Fer1 was the first identified selective small-molecule inhibitor of ferroptosis, and a large number of studies 


\begin{tabular}{|c|c|c|c|c|c|c|}
\hline \multirow{2}{*}{ A $_{\text {Control }}$} & \multirow[b]{2}{*}{ Colitis } & & \multicolumn{4}{|c|}{ I vs Colitis) } \\
\hline & & & Gene & Regulation & Fold Change & $P$ \\
\hline & & $\begin{array}{l}\text { CXI } \\
\text { NOS2 }\end{array}$ & ACSF 2 & Down & 2.4520 & 0.0007 \\
\hline & & CXCL3 & GPX4 & Down & 1.6110 & 0.0032 \\
\hline & & ILIA & LPCAT3 & Down & 1.5058 & 0.0111 \\
\hline & & $\begin{array}{l}I I N G P \\
{ }_{T A P 2}\end{array}$ & NCOA4 & Down & 1.1361 & 0.0446 \\
\hline & & $\begin{array}{l}A C S F 2 \\
G P P A\end{array}$ & ACSL4 & Up & 2.3055 & 0.0390 \\
\hline & & LPCAT3 & SLC38AI & Up & 1.3636 & 0.0236 \\
\hline & & $\begin{array}{l}\text { IRSI } \\
\text { IRSA }\end{array}$ & $G 6 P D$ & Up & 1.3223 & 0.0227 \\
\hline
\end{tabular}
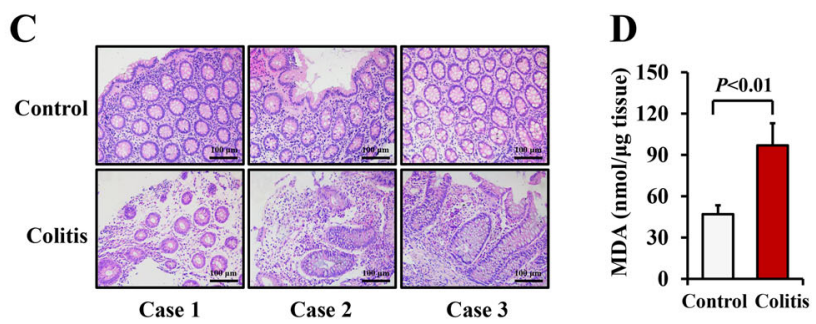

E

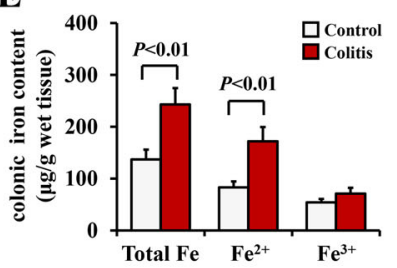

F

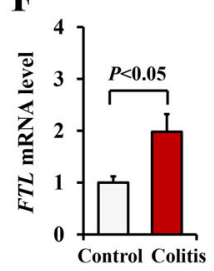

G
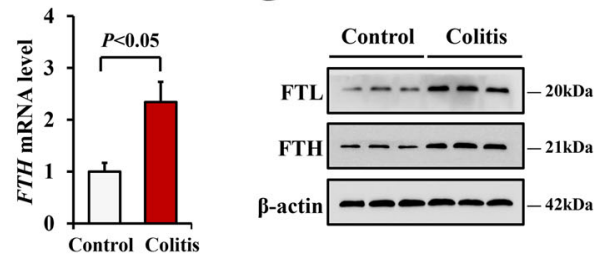

\section{$\mathbf{H}$}

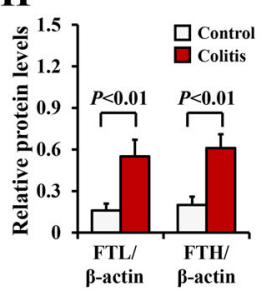

I

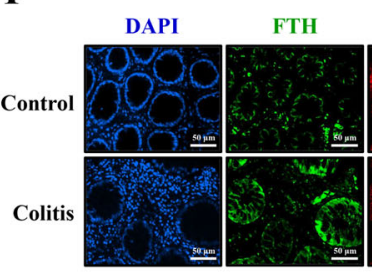

$\mathbf{J}$

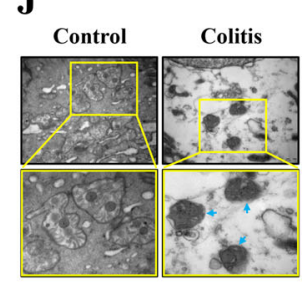

Fig. 1 Ferroptosis was induced in colitis in human. $\mathbf{a}, \mathbf{b}$ Microarray results showed ferroptosis-related genes expression in human colonic biopsy samples from control and ulcerative colitis $(U C)$ patients $(n=3)$. The fold changes in mRNA levels in UC relative to control are represented by green and red squares, showing decreased and increased levels, respectively. The ratio represents the expression value in UC tissues compared with the expression level in control tissues. $P<0.05$ by using Student's $t$ test. c Representative images from H\&E staining of colon tissues from control and UC patients (Scale: $100 \mu \mathrm{m})$. d MDA levels were measured according to MDA Assay Kit. e Iron levels of colonic biopsy tissue were determined by Iron Assay Kit. $\mathbf{f}$ mRNA levels of FTL and FTH were detected by real-time PCR. $\mathbf{g}, \mathbf{h}$ Western blotting analysis of FTL and FTH. $\beta$-actin was used as the loading control. i Double immunofluorescent staining for FTH and cytokeratin 18 (CK 18) were performed in the colonic sections of control and UC patients. Nuclei was stained with DAPI in blue. Localization of FTH was visualized in green and CK 18 was stained in red, the merging positive signals were visualized in yellow (Scale: $50 \mu \mathrm{m}$ ). $\mathbf{j}$ Transmission electron micrographs of colonic epithelial cells from human UC and control samples (Scale: $500 \mathrm{~nm}$ ). Blue arrows indicate shrunken mitochondria. Statistical analyses were performed with Student's $t$ tests.

have verified its efficiency in suppressing ferroptosis both in vivo and in vitro $3,7,25,34$. To determine the role of ferroptosis in colitis, we adopted Fer1 to mice. Interestingly, Fer1 treatment significantly reduced the disease activity score and ameliorated colon length shortening in the experimental colitis mice (Fig. 3a, b). Histological analysis further confirmed the ameliorating effects of Fer1 on colitis, including lessened mucosal erosions and descended inflammatory infiltrations (Fig. 3c, d). These effects were observed after ferroptosis of colonic IECs has inhibited, as necrotic cell death in the colonic IECs from DSS-treated mice was obviously rescued by Fer1 (11.7\% in the DSS + Fer1 group vs $24.0 \%$ in the DSS + NS group) (Fig. 3e). Furthermore, the initial high levels of MDA, iron contents, and FTH were decreased after Fer1 administration (Fig. 3f-h). Deferoxamine (DFO) is another ferroptosis inhibitor that chelates excessive free iron to reduce ferroptosis. Similar to the effects of Fer1, treatment with DFO also alleviated colitis and reduced necrotic cell death of the IECs (Supplementary figure 1). To investigate the survival of the mice, we subjected them to a longer period of $3 \%$ DSS treatment and Fer1 administration. The results showed that Fer1 treatment notably prolong the survival days of mice with colitis (Fig. 3i). Taken together, these results indicated that blockage of ferroptosis by Fer1 ameliorated DSS-induced murine colitis.

\section{Ferroptosis was mediated by ER stress in colitis}

We determined that ferroptosis could be induced in colitis, and it has been reported that ER stress signaling, a vital promotor for UC process, also contributes to the development of ferroptosis ${ }^{14-16}$. In the current study, the levels of proteins involved in the PERK branch of ER stress, including $G$ protein-coupled receptor 78, phosphorylated form of eIF $2 \alpha$ (p-eIF2 $\alpha$ ), ATF4 and CHOP, were elevated in UC samples, a finding in parallel with the increased levels of FTL and FTH (Fig. 4a, b, d, e). We next 


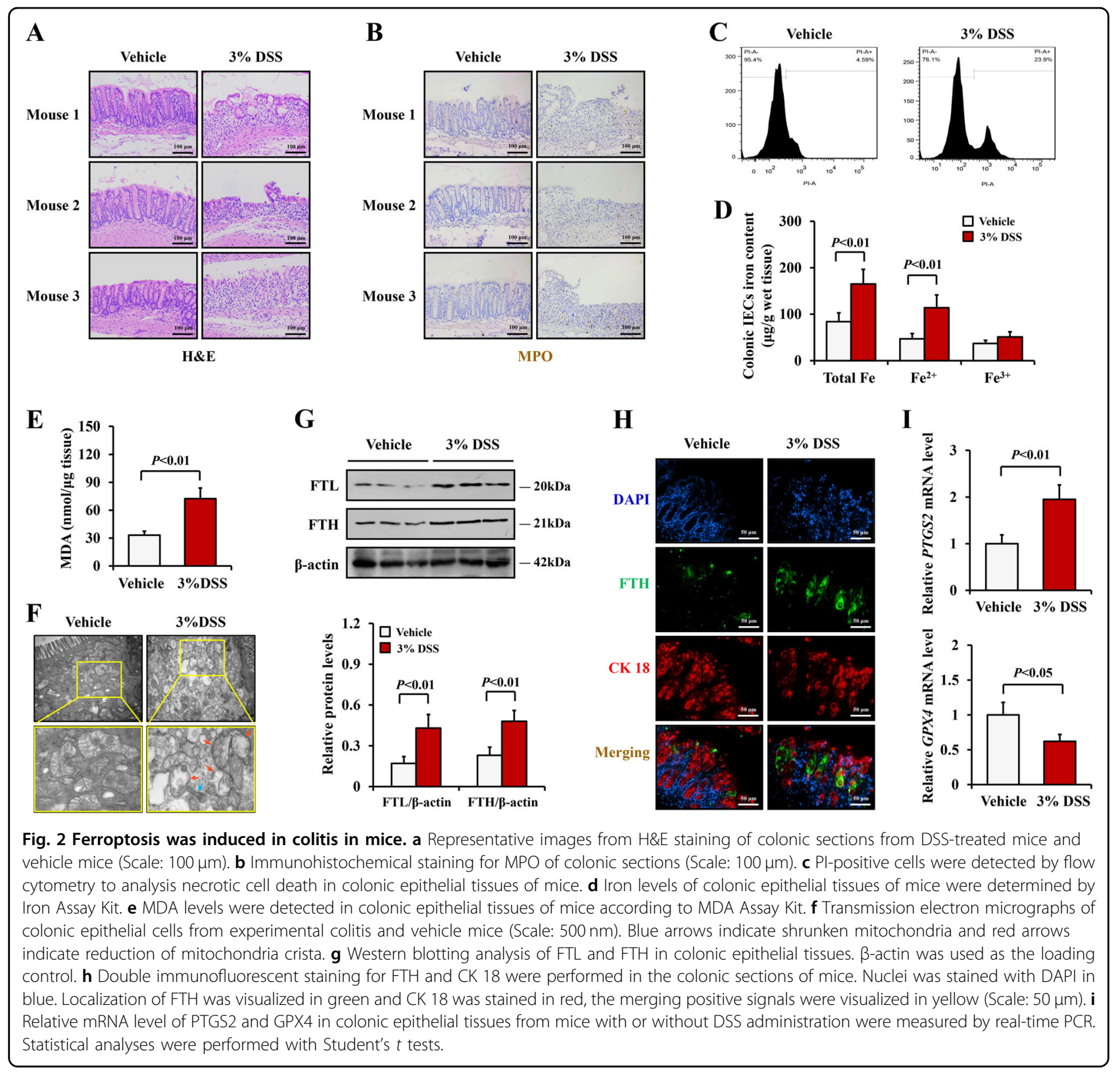

determined whether ER stress was related to IEC ferroptosis. Indeed, evidence obtained by double immunofluorescent staining for p-eIF2 $\alpha$ and FTH revealed that most of the elevated positive signals overlapped in the colonic sections (Fig. 4c, f), suggesting that IEC ferroptosis in colitis might be regulated by ER stress.

To thoroughly define the relationship between ER stress and ferroptosis, $\mathrm{HCoEpiC}$ cells were challenged with RSL3, a canonical inducer of ferroptosis. We observed apparent necrotic cell death and ROS accumulation (Fig. 5a, b) and increased levels of FTL, FTH, and PTGS2 (Fig. 5c, d) in the RSL3-treated cells, which implied that ferroptosis had occurred. In addition, RSL3 administration induced eIF2 $\alpha$ phosphorylation and
ATF4 and CHOP upregulation (Fig. 5c, d). These data indicated that ER stress signaling was involved in RSL3induced ferroptosis. GSK 414 is a selective inhibitor of PERK. The application of GSK 414 in vivo and in vitro effectively suppressed the phosphorylation of PERK and prevented the activation of eIF2 $\alpha /$ ATF $4 / C H O P$ signaling pathway ${ }^{26,27,35}$. When we added GSK 414 to the cells before RSL3 treatment, we were surprised to find that the pretreatment with GSK 414 not only inhibited peIF2 $\alpha$, ATF4, and CHOP expression, but also decreased ferroptosis in the RSL3-stimulated cells (Fig. 5d-g). Together, these investigations suggest an important role for ER stress signaling in the mediation of IEC ferroptosis. 


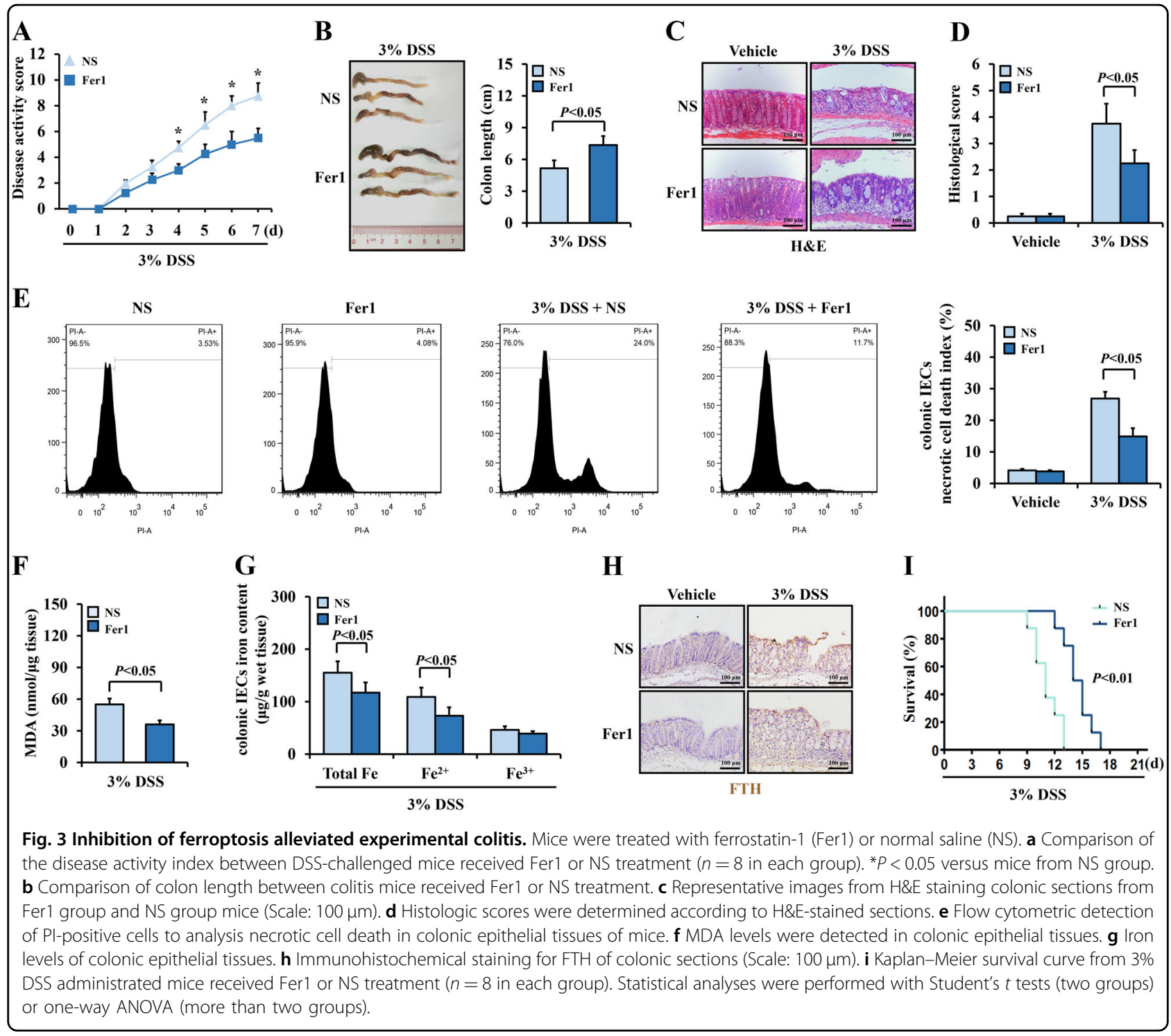

\section{Suppression of ER stress ameliorated colitis via depressing ferroptosis}

On the basis of the observations above, we then assessed whether IEC ferroptosis in colitis mice would be altered when ER stress was suppressed in vivo. Interestingly, the application of GSK 414 obviously ameliorated the disease activity of the colitis mice (Fig. 6a) and alleviated the inflammatory injure of the colonic epithelium (Fig. 6b, c). Similar to the results found in vitro, the inhibition of ER stress in colitis mice led to ferroptosis downregulation. As shown in Fig. 6d-f, h, i, the number of necrotic cell death, the MDA and iron contents and the FTL and FTH protein levels in colonic IECs were all decreased in the GSK 414treated colitis mice. Immunofluorescence detection revealed that the positive signals for p-eIF $2 \alpha$ and FTH in the colon sections from GSK 414-challenged colitis mice were decreased simultaneously as further verified by western blotting (Fig. 6g-i), suggesting that ferroptosis was effectively suppressed by blocking ER stress signaling. Collectively, these evidences confirmed that suppressing ER stress signaling inhibited IEC ferroptosis to ameliorate experimental colitis.

\section{IEC NF-кBp65 exerted protective function against colitis}

After we confirmed that ER stress signaling contributed to IEC ferroptosis in colitis, we explored the possible regulators of this process. Accordingly, NF- $\mathrm{B}$ and ER stress signaling engage in cross-talk under various pathological and physiological conditions ${ }^{22}$. In the gut epithelium, activated NF- $\mathrm{kB}$ exerts protective function against IEC injury and cell death during acute intestinal inflammation. In our study, the immunohistochemistry assay demonstrated that the enhanced p-p65 presented in both the epithelial cells and mesenchymal cells in the 

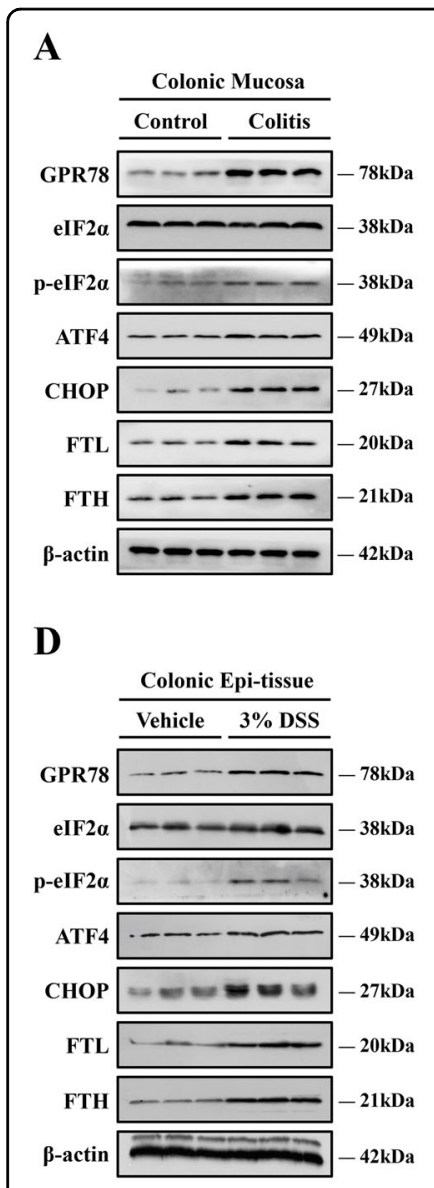
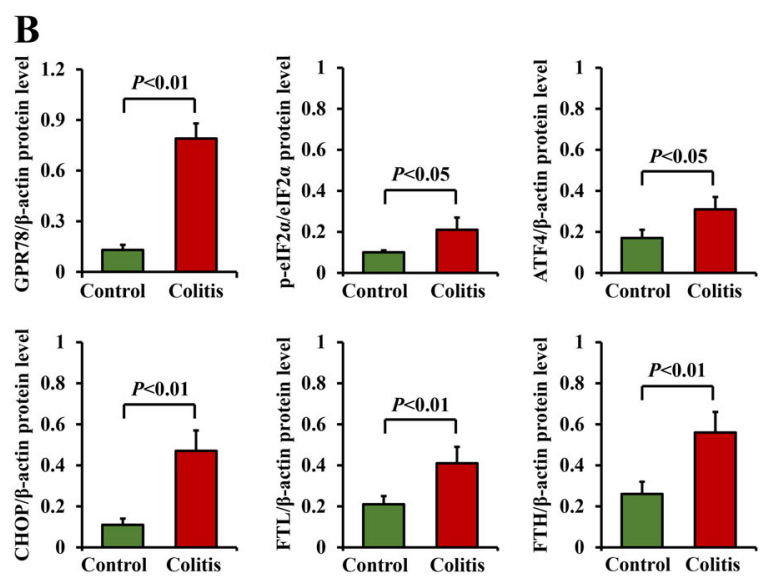

$\mathbf{E}$
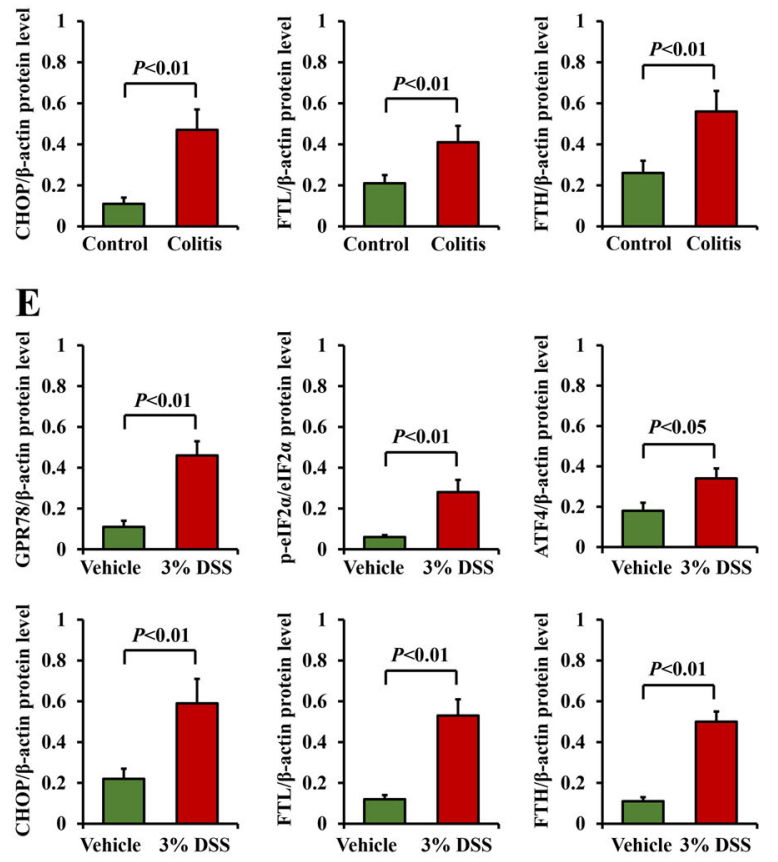

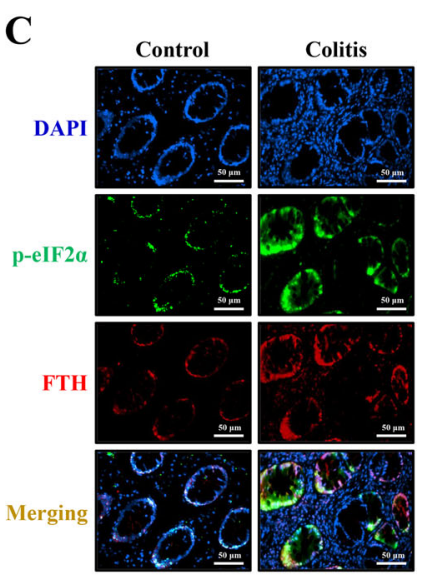

F

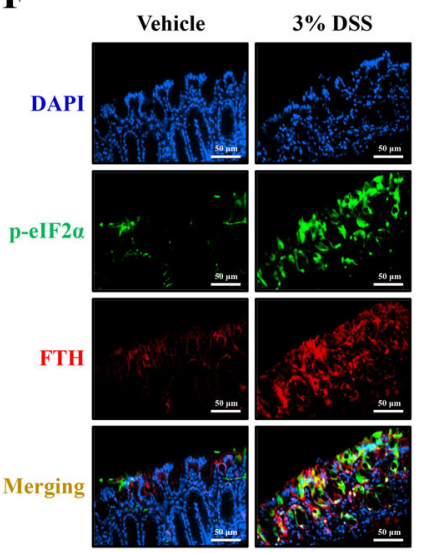

Fig. 4 Ferroptosis was mediated by ER stress in colitis. $\mathbf{a}$, $\mathbf{b}$ Western blotting analysis of ER stress-related proteins and FTL, FTH in colonic mucosal tissue from control and UC patients. c Double immunofluorescent staining for p-elF2a and FTH were performed in the human colonic sections. Nuclei was stained with DAPI in blue. Localization of p-elF2a was stained in green, FTH was visualized in red, and the merging positive signals were visualized in yellow (Scale: $50 \mu \mathrm{m}$ ). d, e Western blotting analysis of ER stress-related proteins and FTL, FTH in colonic epithelial tissue of DSS-treated mice and vehicle group. $\beta$-actin was used as the loading control. f Double immunofluorescent staining for p-elF2a and FTH in colonic sections of mice. Signals were visualized as described in c (Scale: $50 \mu \mathrm{m})$. Statistical analyses were performed with Student's $t$ tests.

colonic sections from UC patients (Fig. 7a). Evidence from the western blot analysis confirmed the elevation of p-p65 in the mucosal tissue samples from the UC patients (Fig. 7b). Similar results were observed in the colonic sections or colonic epithelial tissues of the experimental colitis mice (Fig. 7c, d).

To clarify the function of IEC NF-kBp65 in colitis, we ablated the p65 gene specially in mouse IECs (p65 ${ }^{\mathrm{IEC}-\mathrm{KO}}$ ). As measured by double immunofluorescence assay, the signal of p65 vanished in the intestinal epithelium from the $065^{\mathrm{IEC}-\mathrm{KO}}$ mice (Fig. 7e). After DSS challenge, the p $65^{\mathrm{IEC}-\mathrm{KO}}$ mice presented higher disease activity and shorter colon lengths on day 7 than were presented by WT mice (Fig. 7f-h). Furthermore, histopathologic analysis revealed that the deficiency of NF-kBp65 in the IECs led to more severe epithelial destruction and intestinal inflammation in colitis mice (Fig. 7i, j). Finally, p65 $5^{\mathrm{IEC}-\mathrm{KO}}$ mice had remarkably fewer survival days than their WT littermates when consecutive treated with DSS (Fig. 7k). These data collectively implied that the activated NFКBp65 in IECs had protective function against DSSinduced inflammation-related injury.

\section{Deficiency of IEC NF-KBp65 led to upregulated ER stress- mediated ferroptosis in colitis}

Given that activated NF- $k B p 65$ protected IECs from inflammatory injury in colitis and that NF- $\mathrm{KB}$ is thought to be involved in the regulation of ER stress and ferroptosis process, we conjectured that NF- $\mathrm{BP} 65$ might regulate ER stress-mediated ferroptosis. As expected, DSS-treated p $65^{\mathrm{IEC}-\mathrm{KO}}$ mice displayed a markedly higher percentage of necrotic cell death in colonic IECs (35.2\% in DSS-treated p $65^{\mathrm{IEC}-\mathrm{KO}}$ mice vs $24.5 \%$ in DSS-treated WT mice) and higher levels of MDA and iron compared with that in the WT mice (Fig. 8a-c). Interestingly, the increased necrotic cell death, MDA, and iron levels in DSS-challenged p65 $5^{\mathrm{IEC}-\mathrm{KO}}$ 


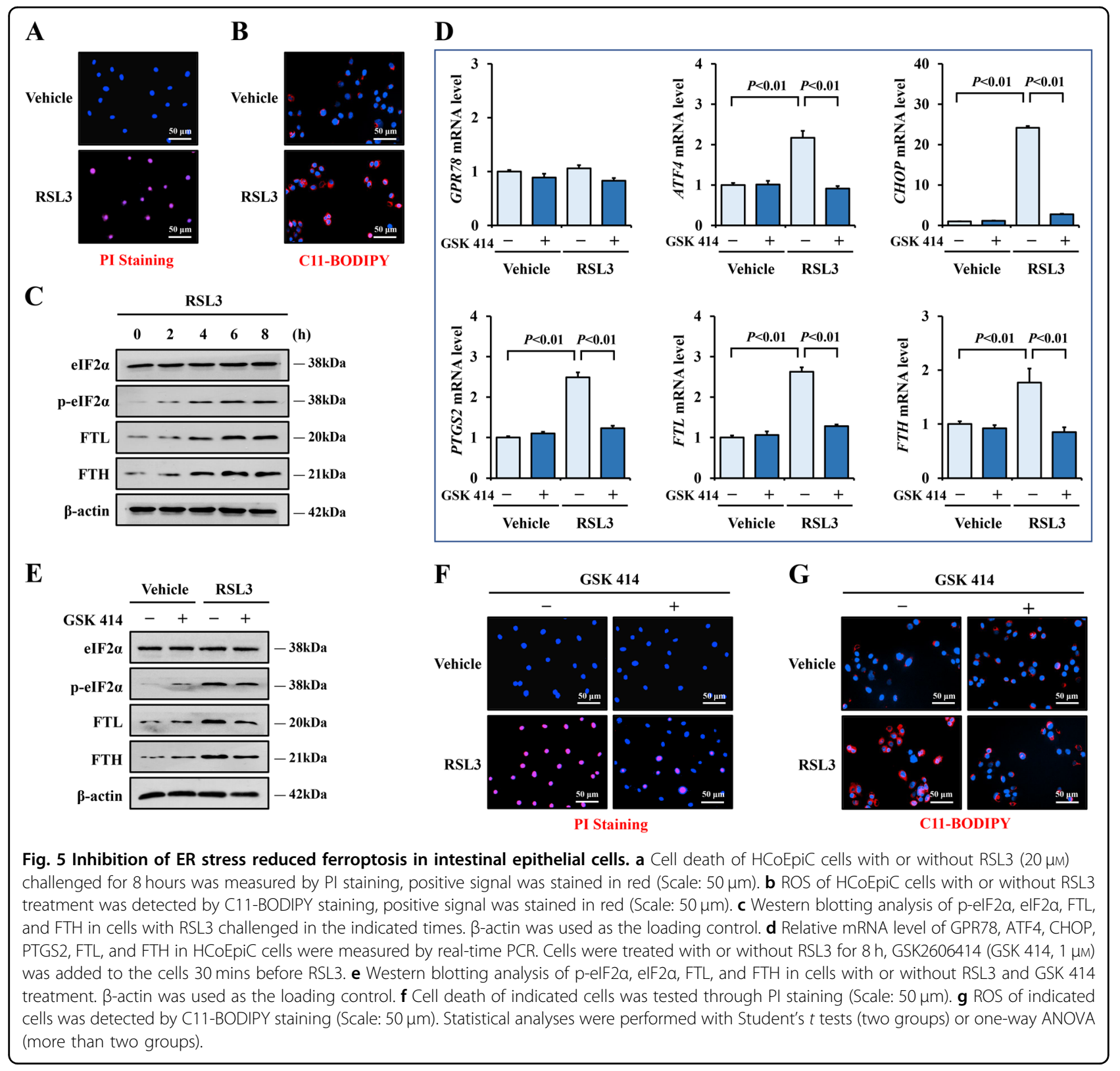

mice could be thoroughly rescued by Fer1 treatment (Supplementary figure 2). These data suggested a suppressive function of IEC-derived NF-kBp65 on ferroptosis. As ferroptosis of the IECs was mediated by ER stress, we proposed that NF- $\mathrm{kBp} 65$ rescued ferroptosis by inhibiting ER stress signaling. Indeed, $\mathrm{p} 65^{\mathrm{IEC}-\mathrm{KO}}$ mice had increased levels of p-eIF2 $\alpha$ and ferritin proteins after DSS administration (Fig. $8 \mathrm{~d}, \mathrm{e}$ ), and the double immunofluorescence further showed that the p-eIF2 $\alpha$ and FTH signatures were increased synchronously (Fig. 8f-h), indicating a very promising function of NF-kBp65 in suppressing ER stress/ ferroptosis signaling in IECs. In brief, these data confirmed that NF-kBp65 deficiency in the IECs exacerbated ER stress-mediated ferroptosis in experimental colitis.

\section{Phosphorylation of NF-KBp65 inhibited ER stress by interacting with elF2a}

Next, we sought to determine how NF-kBp65 regulated ER stress signaling. We treated HCoEpiC cells with TNF- $\alpha$, a canonical activator of NF- $\mathrm{kB}$. The TNF- $\alpha$ administration increased the levels of $\mathrm{p}-\mathrm{p} 65$, and simultaneously induced the phosphorylation of eIF2 $\alpha$ (Fig. 8i). Immunofluorescent staining showed few positive p-p65 signals and negligible levels of p-eIF $2 \alpha$ in the cells under physiological conditions, but abundant and overlapping positive signals of p-p65 and p-eIF2 $\alpha$ were observed in the cells treated with TNF- $\alpha$ (Fig. 8j). To confirm the mechanism of the p65 and eIF2 $\alpha$ interaction, we conducted a coimmunoprecipitation experiment. The results indicated that p65 interacted with 


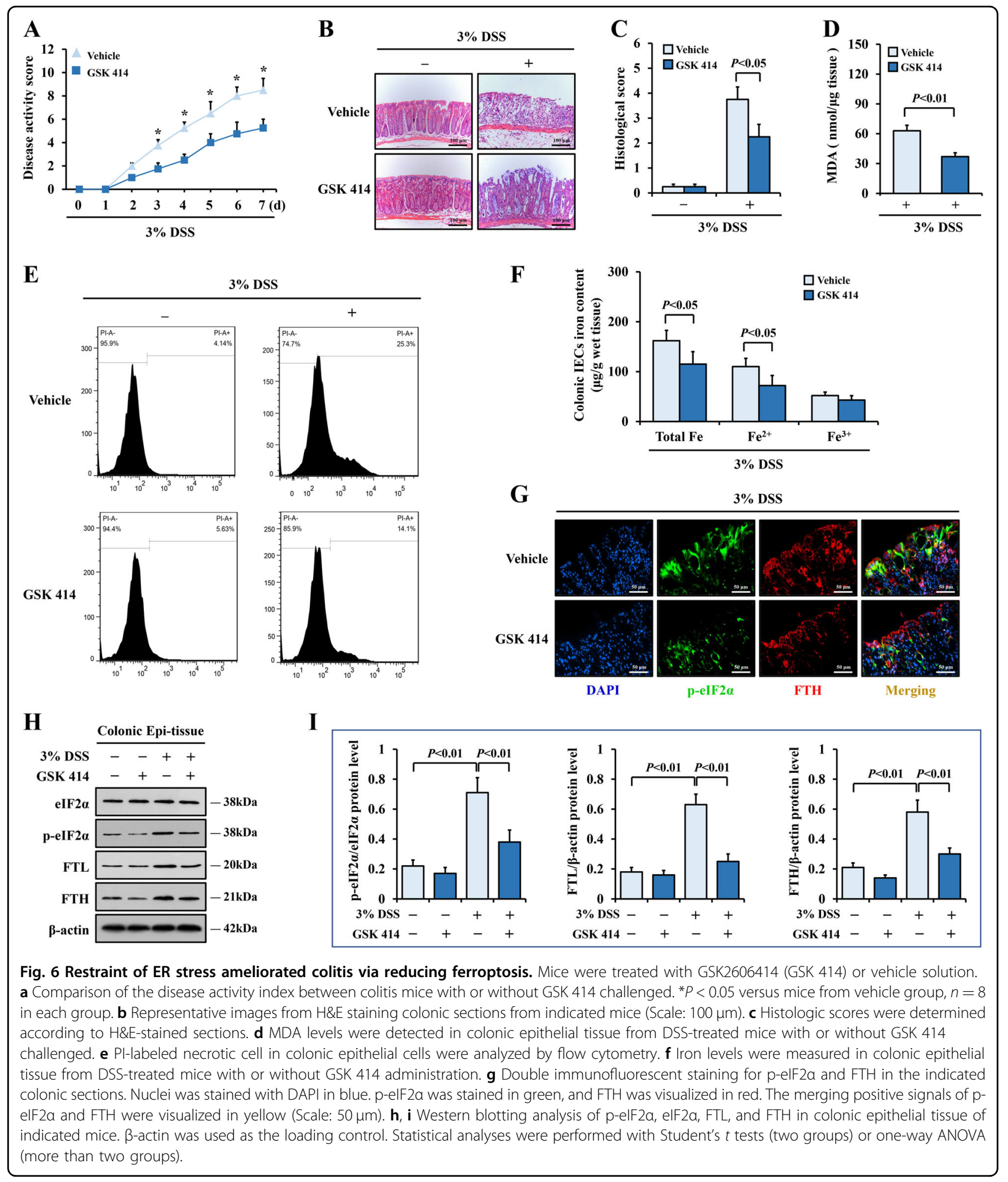

eIF2 $\alpha$ by binding to it. (Fig. 8k). Surprisingly, we observed that inhibiting the activation of NF-kBp65 by BAY 11-7085 led to enhanced expression of p-eIF $2 \alpha$ under both normal conditions and TNF- $\alpha$ challenge (Fig. 81), indicating that phosphorylation of p65 exerted a suppressive function on eIF2 $\alpha$ activation. These results demonstrated the critical role of activated NF-kBp65 in inhibiting ER stress signaling through its interaction with eIF2 $\alpha$. 


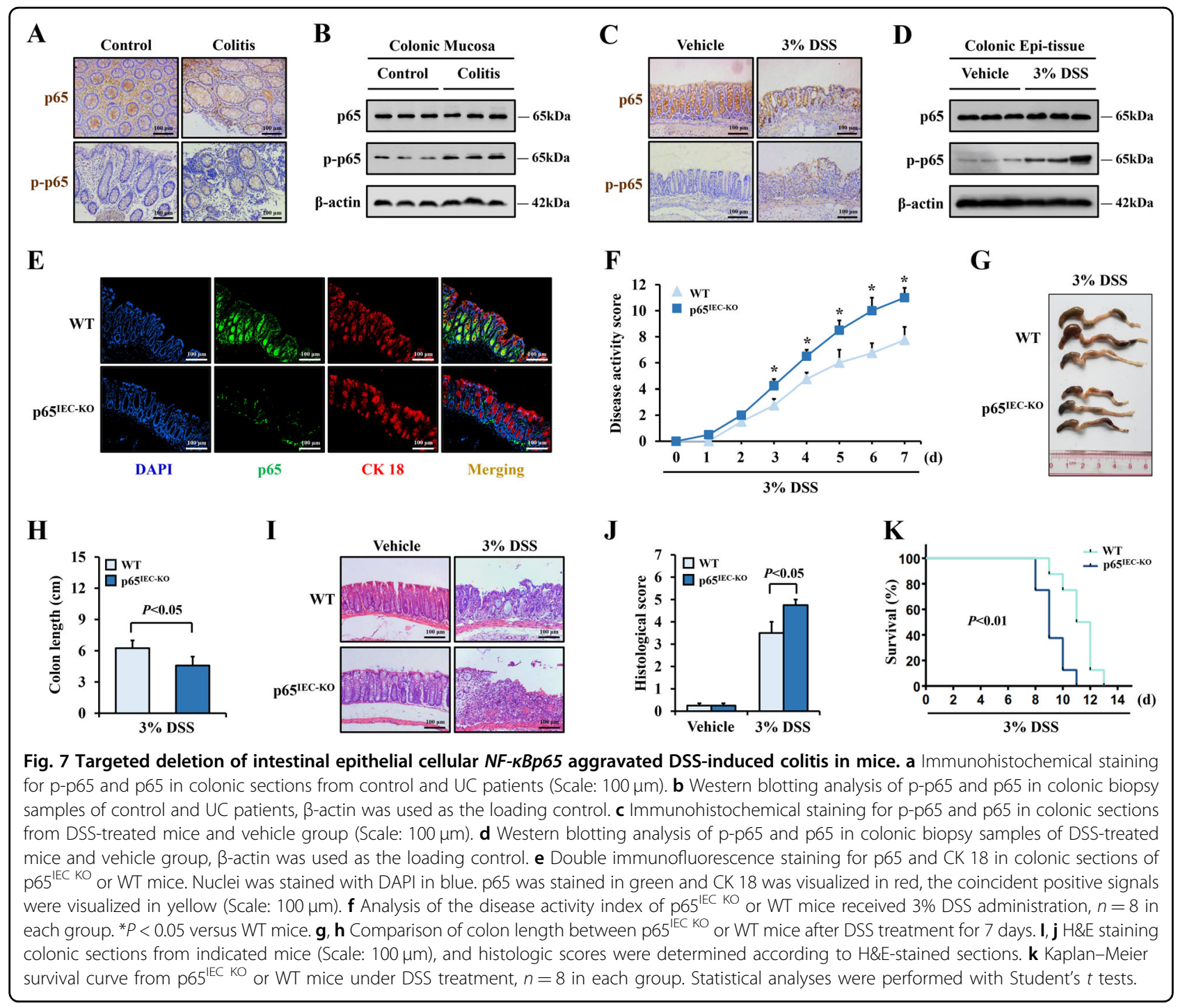

\section{Discussion}

UC is an inflammatory-associated disease characterized by persistent damage to epithelial cells of the colon. So far, the mechanism of UC pathogenesis has not been completely understood. Herein, we first proposed that iron- and ROSdependent ferroptosis involves in colonic epithelial cell death of UC. More importantly, we revealed that IEC ferroptosis in UC was mediated via ER stress signaling and primarily suppressed by phosphorylated-NF-kBp65.

In recent decades, existing studies have reported that iron and ROS contribute to UC development ${ }^{9,10}$. However, the detailed injured mechanism of iron and ROS has not been well described. Ferroptosis is a novel form of regulated necrosis that triggered by excess ferrous iron and accumulated $\mathrm{ROS}^{3}$. Our data showed that several ferroptosis-associated gene transcripts notably altered in UC specimens. Shrunken mitochondria, increased necrotic cell death, and upregulated iron and ROS in colonic mucosa/IECs were observed in human UC and DSS-induced murine colitis. These data suggested that ferroptosis was a cause of colitis-associated IEC cell death as was further verified through the administration of Fer1, a specific inhibitor of ferroptosis, to the mice.

ER stress is well described as a significant modulator of inflammatory damage and even triggers cell death if it persistent exists ${ }^{36}$. Genetic abnormalities and aberrant environmental factors of the gut account for the induction of ER stress in IECs of UC. A genetic study revealed primary aberrance in several ER homeostasis-associated genes, including $A G R 2, X B P 1$, and $O R M D L 3$ in $\mathrm{UC}$ patients ${ }^{37}$. The exposure to high levels of inflammatory cytokines such as TNF- $\alpha$ disturbs protein folding function of ER and alters ER homeostasis in IECs ${ }^{38,39}$. Moreover, various microbiota and microbial metabolites in the colonic lumen of UC activate immunity response and further trigger IEC ER stress ${ }^{40}$. Our previous study 


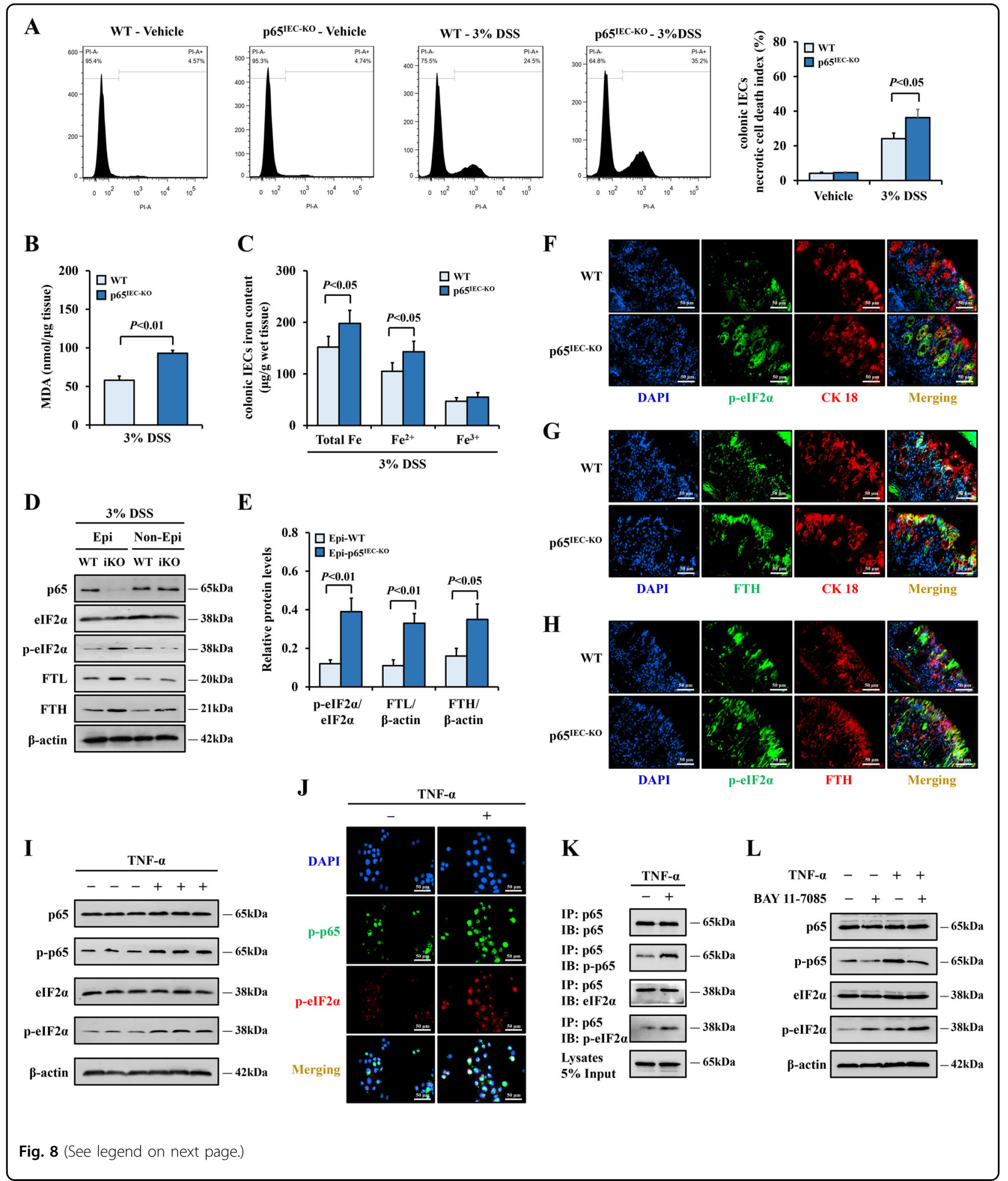

demonstrated that ER stress-mediated colonic IEC apoptosis in $\mathrm{UC}^{17}$. The current data showed that eIF2 $\alpha /$ ATF4/CHOP signaling was upregulated in UC-derived colonic IECs. Interestingly, we also determined that ER stress participated in the development of ferroptosis because signatures of ER stress and ferroptosis overlapped mainly in the colonic IECs of UC. ER stress-facilitated ferroptosis has been implicated in previous researches. 
(see figure on previous page)

Fig. 8 Phosphorylation of intestinal epithelial NF-KBp65 inhibited ER stress-mediated ferroptosis in colitis via interacting with elF2a. a Necrotic cell death in colonic epithelial tissues from p65 $5^{\mathrm{IEC}-\mathrm{KO}}$ or WT mice was labeled by PI and analyzed through flow cytometry. $\mathbf{b}, \mathbf{c}$ MDA levels and iron levels were detected in colonic epithelial tissues from p65 $5^{\mathrm{IEC}-\mathrm{KO}}$ or WT mice received DSS administration. $\mathbf{d}$, e Western blotting analysis of p65, p-elF2a, elF2a, FTL, and FTH in colonic epithelial/non-epithelial tissues from p65 $5^{\mathrm{IEC}} \mathrm{KO}$ or WT mice after DSS treatment. $\beta$-actin was used as the loading control. $\mathbf{f}$ Double immunofluorescence staining for $p$-elF2a and CK 18 in colonic sections of p65 ${ }^{\mathrm{IEC}}$ KO or WT mice with DSS challenged. Nuclei was stained with DAPI in blue. p-elF2a was stained in green and CK 18 was visualized in red, the coincident positive signals were visualized in yellow (Scale: $50 \mu \mathrm{m})$. $\mathbf{g}$ Double immunofluorescence staining for FTH and CK 18 in colonic sections. FTH was stained in green, and CK 18 was visualized in red, the merging positive signals were visualized in yellow (Scale: $50 \mu \mathrm{m}$ ). $\mathbf{h}$ Double immunofluorescence staining for p-elF2a and FTH in colonic sections. p-elF2a was stained in green and FTH was visualized in red, the coincident positive signals were visualized in yellow (Scale: $50 \mu \mathrm{m})$. i Western blotting of p-p65, p65 p-elF2a, and elF2a in HCoEpiC cells with or without TNF-a treatment ( $40 \mathrm{ng} / \mathrm{ml}, 1 \mathrm{~h}$ ). $\beta$-actin was used as the loading control. $\mathbf{j}$ Immunofluorescence staining for p-p65 and p-elF2a in HCoEpiC cells. Nuclei was stained with DAPI in blue. p-p65 was stained in green and p-elF2a was visualized in red, the overlapped positive signals were visualized in yellow (Scale: $50 \mu \mathrm{m}$ ). $\mathbf{k} \mathrm{HCoEpiC}$ cells with or without TNF-a challenged were subjected to immunoprecipitation with an anti-p65 antibody. Co-immunoprecipitated endogenous p-p65, elF2a, and p-elF2a were detected with corresponding antibodies. IP immunoprecipitate, IB immunoblot. I Analysis of the effect of BAY 11-7085 (10 $\mu \mathrm{m}$, adding to the indicated wells 15 mins before TNF-a administration) on the activation of p65 and elF2a in cells with or without TNF-a treatment through western blotting. $\beta$-actin was used as the loading control. Statistical analyses were performed with Student's $t$ tests (two groups) or one-way ANOVA (more than two groups).

Park, et al. ${ }^{14}$ found that whole cigarette smoke condensates induce ferroptosis in bronchial epithelial cells via ER stress. To verify this mechanism, we administered GSK 414, a specific inhibitor of the PERK branch of ER stress signaling both in vivo and in vitro. It came out that GSK 414 treatment clearly downregulated IEC necrotic cell death, iron contents, and ROS in the mice with colitis. Moreover, in ferroptosis inducer RSL3-challenged HCoEpiC cells, pretreatment with GSK 414 notably rescued cells from ferroptosis. These data are consistent with the results from the studies described above and indicate a new role for ER stress, showing that it contributes not only to apoptosis but also to ferroptosis in the IECs of UC. Nevertheless, our results did not clarify the concrete regulatory mechanism of ER stress on ferroptosis. Therefore, further studies are needed.

Interestingly, accumulating evidence indicates a protective role of NF- $\mathrm{kB}$ in the IECs, promoting resistance to damage and cell death. Our previous study revealed that blocking the activity of NF- $\mathrm{kB}$ with BAY 11-7085 in vivo aggravated IECs apoptosis in $\mathrm{UC}^{20}$. Other studies have found that mice with gene deletion of NF- $\mathrm{kB}$ member were more susceptible to inflammatory damage and death ${ }^{41,42}$. In addition, NF- $\mathrm{KB}$ is a critical regulator for both ER stress signaling and ferroptosis process ${ }^{21,22}$. Consistent with previous literature, the current data showed that mice lacking NF-kBp65 in IECs led to more serious DSS-induced colitis. Interestingly, our study also provides exquisite evidence that the deletion of IEC NF-kBp65 in mice caused upregulated IEC necrotic cell death and increased levels of ferrous iron and ROS in colitis, implicating that NF-kBp65 resisted IEC ferroptosis in colitis. Noteworthily, our further results elucidated that NF-kBp65 suppressed ferroptosis via downregulation of ER stress, as DSS-treated $\mathrm{p} 65^{\mathrm{IEC}-\mathrm{KO}}$ mice displayed synchronous elevation of p-eIF2 $\alpha$ and FTH in colonic epithelial cells. These observations provide a new perspective for recognizing IEC NF-kBp65 against ER stress-mediated ferroptosis in UC.
We further clarified how NF-kBp65 regulates ER stressassociated ferroptosis. Through coimmunoprecipitation in HCoEpiC cells, NF-kBp65 was found to inhibit ER stressmediated ferroptosis via direct interaction with eIF2 $\alpha$, a key component of the PERK branch of ER stress. The application of BAY 11-7085 in cells confirmed that activated NF-kBp65 exerted a suppressive function on the phosphorylation of eIF2 $\alpha$. This result was different from that of previous reports, indicating that NF- $\mathrm{kB}$ can be activated by ER stress signaling and that eIF2 $\alpha$ acts as a direct upstream mediator for NF- $\mathrm{kB}$ activation $^{20,43-45}$. Herein, ER stress inducer thapsigargin$(4 \mu \mathrm{M})$ and tunicamycin- $(10 \mu \mathrm{g} / \mathrm{ml})$ treated HCoEpiC cell presented enhanced level of p-p65, which could be blocked by GSK 414 (data not shown). These results are consistent with that from previous literature ${ }^{43,44}$. However, when we pretreated the cells with BAY 11-7085 to inhibit the activity of p65 before thapsigargin/tunicamycin administration, the phosphorylation of eIF2 $\alpha$ was enhanced (data not shown), a finding that was similar to the data shown in Fig. 8l. These observations indicated a novel relationship between ER stress and NF-kB, suggesting a feedback loop between them. Generally speaking, the induction of ER stress can activate NF-kBp65, and phosphorylated-NF-kBp65 produces negative feedback on ER stress and subsequently suppresses ER stress-mediated ferroptosis.

Conclusively, our findings revealed that ferroptosis contributes to IEC cell death in UC and that ER stress signaling mediates IEC ferroptosis. Phosphorylated-NF-kBp65 suppresses ER stress-mediated IEC ferroptosis by interacting with eIF2 $\alpha$. These data suggest that inhibiting ferroptosis will be a new therapeutic method and that the phosphorylation of NF- $\mathrm{kBp} 65$ is a potential therapeutic target for UC.

\section{Acknowledgements}

We thank Professor Jianping Ye at Pennington Biomedical Research Center, Louisiana State University System, Baton Rouge, LA, USA, for providing mice carrying loxP-flanked NF-KBp65 allele. This work was supported by the grants from the National Natural Science Foundation of China (grant numbers U1501224, 81800458, 81700536), the Natural Science Foundation Team Project 
of Guangdong Province (grant numbers 2018B030312009), the Science and Technology Developmental Special Foundation of Guangdong Province (grant numbers 2017B020226003).

\section{Conflict of interest}

The authors declare that they have no conflict of interest.

\section{Publisher's note}

Springer Nature remains neutral with regard to jurisdictional claims in published maps and institutional affiliations.

Supplementary Information accompanies this paper at (https://doi.org/ 10.1038/s41419-020-2299-1).

Received: 14 November 2019 Revised: 23 January 2020 Accepted: 23 January 2020

Published online: 03 February 2020

\section{References}

1. Ungaro, R., Mehandru, S., Allen, P. B., Peyrin-Biroulet, L. \& Colombel, J. F. Ulcerative colitis. Lancet 389, 1756-1770 (2017).

2. de Souza, H. S. \& Fiocchi, C. Immunopathogenesis of IBD: current state of the art. Nat. Rev. Gastroenterol. Hepatol. 13, 13-27 (2016).

3. Dixon, S. J. et al. Ferroptosis: an iron-dependent form of nonapoptotic cell death. Cell 149, 1060-1072 (2012).

4. Xie, Y. et al. Ferroptosis: process and function. Cell Death Differ. 23, 369-379 (2016).

5. Yang, W. S. et al. Regulation of ferroptotic cancer cell death by GPX4. Cell 156, 317-331 (2014)

6. Friedmann Angeli, J. P. et al. Inactivation of the ferroptosis regulator Gpx4 triggers acute renal failure in mice. Nat. Cell Biol. 16, 1180-1191 (2014).

7. Skouta, R. et al. Ferrostatins inhibit oxidative lipid damage and cell death in diverse disease models. J. Am. Chem. Soc. 136, 4551-4556 (2014).

8. Yang, W. S. \& Stockwell, B. R. Ferroptosis: death by lipid peroxidation. Trends Cell Biol. 26, 165-176 (2016).

9. Millar, A. D. Rampton, D. S. \& Blake, D. R. Effects of iron and iron chelation in vitro on mucosal oxidant activity in ulcerative colitis. Aliment. Pharm. Ther. 14, 1163-1168 (2000).

10. Minaiyan M., Mostaghel E., Mahzouni P. Preventive therapy of experimental colitis with selected iron chelators and anti-oxidants. Int. J. Prev. Med. 3 S162-S169 (2012)

11. Seril, D. N. et al. Dietary iron supplementation enhances DSS-induced colitis and associated colorectal carcinoma development in mice. Dig. Dis. Sci. 47, 1266-1278 (2002).

12. Kobayashi, $Y$, et al. Association between dietary iron and zinc intake and development of ulcerative colitis: a case-control study in Japan. J. Gastroenterol. Hepatol. 34, 1703-1710 (2019).

13. Yoshida, H. ER stress and diseases. FEBS J. 274, 630-658 (2007).

14. Park, E. J., Park, Y. J., Lee, S. J., Lee, K. \& Yoon, C. Whole cigarette smoke condensates induce ferroptosis in human bronchial epithelial cells. Toxicol. Lett. 303, 55-66 (2019).

15. Dixon, S. J. et al. Pharmacological inhibition of cystine-glutamate exchange induces endoplasmic reticulum stress and ferroptosis. Elife $\mathbf{3}$, e02523 (2014)

16. Lee, Y. S., Lee, D. H., Choudry, H. A., Bartlett, D. L. \& Lee, Y. J. Ferroptosis-induced endoplasmic reticulum stress: cross-talk between ferroptosis and apoptosis. Mol. Cancer Res. 16, 1073-1076 (2018).

17. Zeng, L. X. et al. $\beta$-Arrestin2 encourages inflammation-induced epithelial apoptosis through ER stress/PUMA in colitis. Mucosal Immunol. 8, 683-695 (2015).

18. Pasparakis, M. Regulation of tissue homeostasis by NF-kappaB signalling: implications for inflammatory diseases. Nat. Rev. Immunol. 9, 778-788 (2009).

19. McDaniel, D. K., Eden, K., Ringel, V. M. \& Allen, I. C. Emerging roles for noncanonical NF-KB signaling in the modulation of inflammatory bowel disease pathobiology. Inflamm. Bowel Dis. 22, 2265-2279 (2016).
20. Qiu, W. et al. PUMA-mediated intestinal epithelial apoptosis contributes to ulcerative colitis in humans and mice. J. Clin. Invest. 121, 1722-1732 (2011).

21. Chen, P. et al. Kinome screen of ferroptosis reveals a novel role of ATM in regulating iron metabolism. Cell Death Differ. https://doi.org/10.1038/s41418019-0393-7 (2019).

22. Schmitz, M. L., Shaban, M. S., Albert, B. V., Gökçen, A. \& Kracht, M. The crosstalk of endoplasmic reticulum (ER) stress pathways with NF-kb: complex mechanisms relevant for cancer, inflammation and infection. Biomedicines $\mathbf{6}$, 58 (2018).

23. Annese, $V$. et al. European evidence based consensus for endoscopy in inflammatory bowel disease. J. Crohns Colitis 7, 982-1018 (2013).

24. Yang, Y. et al. $\beta$-Arrestin1 enhances hepatocellular carcinogenesis through inflammation-mediated Akt signalling. Nat. Commun. 6, 7369 (2015).

25. Wang, $\mathrm{H}$. et al. Characterization of ferroptosis in murine models of hemochromatosis. Hepatology 66, 449-465 (2017).

26. Axten, J. M. et al. Discovery of 7-methyl-5-(1-\{[3-(trifluoromethyl)phenyl]acety $\}$ 2,3-dihydro-1H-indol-5-yl)-7H-pyrrolo[2,3-d]pyrimidin-4-amine (GSK2606414), a potent and selective first-in-class inhibitor of protein kinase R (PKR)-like endoplasmic reticulum kinase (PERK). J. Med. Chem. 55, 7193-7207 (2010).

27. Moreno, J. A. et al. Oral treatment targeting the unfolded protein response prevents neurodegeneration and clinical disease in prion-infected mice. Sci. Transl. Med. 5, 206 ra138 (2013).

28. Tüzün, A. et al. Oxidative stress and antioxidant capacity in patients with inflammatory bowel disease. Clin. Biochem. 35, 569-572 (2002).

29. Biasi, F., Leonarduzzi, G., Oteiza, P. I. \& Poli, G. Inflammatory bowel disease: mechanisms, redox considerations, and therapeutic targets. Antioxid. Redox Signal. 19, 1711-1747 (2013).

30. Yang, L. et al. Difference in pathomechanism between Crohn's disease and ulcerative colitis revealed by colon transcriptome. Inflamm. Bowel Dis. $\mathbf{2 5}$ 722-731 (2019).

31. Mirkov, M. U., Verstockt, B. \& Cleynen, I. Genetics of inflammatory bowel disease: beyond NOD2. Lancet Gastroenterol. Hepatol. 2, 224-234 (2017).

32. Gao, M. et al. Ferroptosis is an autophagic cell death process. Cell Res. $\mathbf{2 6}$ 1021-1032 (2016).

33. Andrews, N. C. \& Schmidt, P. J. Iron homeostasis. Annu Rev. Physiol. 69, 69-85 (2007).

34. Linkermann, A. et al. Synchronized renal tubular cell death involves ferroptosis. Proc. Natl Acad. Sci. USA 111, 16836-16841 (2014).

35. Yan, F. et al. Pharmacological inhibition of PERK attenuates early brain injun after subarachnoid hemorrhage in rats through the activation of Akt. Mol. Neurobiol. 54, 1808-1817 (2017).

36. Zhao, L. \& Ackerman, S. L. Endoplasmic reticulum stress in health and disease. Curr. Opin. Cell Biol. 18, 444-452 (2006).

37. Maloy, K. J. \& Powrie, F. Intestinal homeostasis and its breakdown in inflammatory bowel disease. Nature 474, 298-306 (2011).

38. Xue, X. et al. Tumor necrosis factor alpha (TNFalpha) induces the unfolded protein response (UPR) in a reactive oxygen species (ROS)-dependent fashion, and the UPR counteracts ROS accumulation by TNFalpha. J. Biol. Chem. 280, 33917-33925 (2005)

39. $\mathrm{Ma}, \mathrm{X}$. et al. Intestinal epithelial cell endoplasmic reticulum stress and inflammatory bowel disease pathogenesis: an update review. Front Immunol. 8, 1271 (2017).

40. Kaser, A. \& Blumberg, R. S. Endoplasmic reticulum stress and intestinal inflammation. Mucosal Immunol. 3, 11-16 (2010).

41. Steinbrecher, K. A., Harmel-Laws, E., Sitcheran, R. \& Baldwin, A. S. Loss of Epithelial RelA Results in Deregulated Intestinal Proliferative/Apoptotic Homeostasis and Susceptibility to Inflammation. J. Immunol. 180, 2588-2599 (2008).

42. Nenci, A. et al. Epithelial NEMO links innate immunity to chronic intestinal inflammation. Nature 446, 557-561 (2007).

43. Jiang, H. Y. et al. Phosphorylation of the a subunit of eukaryotic initiation factor 2 is required for activation of NF-KB in response to diverse cellular stresses. Mol. Cell Biol. 23, 5651-5663 (2003).

44. Deng, J. et al. Translational repression mediates activation of nuclear factor kappa B by phosphorylated translation initiation factor 2. Mol. Cell Biol. 24 10161-10168 (2004)

45. Chen J., Stark L. A. Crosstalk between NF-kB and nucleoli in the regulation of cellular homeostasis. Cells. 7, E157 (2018). 